\title{
Estimates Related to the Extended Spectral Control of the Wave Equation
}

\author{
Cheikh Seck ${ }^{1,2}$, Abdoulaye Sène ${ }^{3} \&$ Mary Teuw Niane ${ }^{1}$ \\ ${ }^{1}$ Laboratoire d'Analyse Numérique et d'Informatique, BP 234, Université Gaston Berger, Saint-Louis, Sénégal \\ 2 Département de Mathématiques de la FASTEF, Université Cheikh Anta Diop, Dakar, Sénégal \\ ${ }^{3}$ Département de mathématiques, Facultè des Sciences, Université Cheikh Anta Diop, Dakar, Sénégal \\ Correspondence: Cheikh Seck, Département de Mathématiques de la FASTEF ex ENS de l'Université Cheikh Anta Diop \\ de Dakar, Sénégal. Tel: (+221) 776512735. E-mail: cheikh5.seck@ucad.edu.sn/cseckdiakhao@gmail.com
}

Received: May 15, 2018 Accepted: May 30, 2018 Online Published: July 25, 2018

doi:10.5539/jmr.v10n4p156

URL: https://doi.org/10.5539/jmr.v10n4p156

\section{Abstract}

In this work, we show through a numerical approach the extended spectral controllability of the wave equation over a portion of a regular domain, and that the spectrum obtained is quasi-symmetrical. Numerical simulations also verify the analytical estimates demonstrated in Niane and al. [4] and the graphic illustrations relating to this spectral controllability were done.

Keywords: Control, spectral controllability, norm majorations, numerical simulations

\section{Introduction}

\subsection{Introduce the Problem}

For the wave equation in Niane and al. [4], the authors has been proved the existence of a $C^{\infty}$-control with a support in an open portion $\omega$ of the domain $\Omega$ as small as they wish control a finite number of uncontrolled eigenmodes. Furthermore the disruption on other modes is inversely proportional to the control duration. Thus, the estimates related to the spectral controllability have been analytically proven for the wave equation.

In the sequel, let $\Omega$ be a bounded smooth domain of $\mathbb{R}^{2}, T>0$ the maximum observability time and $\omega$ an open subset of $\Omega$. We consider the wave equation with state $\phi$

$$
\left\{\begin{array}{c}
\left.\phi^{\prime \prime}-\Delta \phi=v \cdot \chi_{\omega} \text { in } Q_{T}=\Omega \times\right] 0, T[ \\
\left.\phi(t)=0 \text { on } \Gamma_{T}=\Gamma \times\right] 0, T[ \\
\phi(0)=\phi_{0} \\
\phi^{\prime}(0)=\phi_{1}
\end{array}\right.
$$

where $\phi_{0} \in H_{0}^{1}(\Omega)$ and $\phi_{1} \in L^{2}(\Omega)$.

\subsection{Explore Importance of the Problem}

The problem of exact internal controllability of the wave equation is to find a control $v \in L^{2}\left(0, T ; L^{2}(\omega)\right)$ such that if $\phi$ is the solution of (1), then $\phi(T)=\phi^{\prime}(T)=0$.

Existence results were already established from the HUM method Lions [6, 7], and Niane [4].

Thus, we know that three major difficult achievements of these controls appear:

- If $\omega$ is too widely open as support controls.

- If $\omega$ is too small on the usual functional spaces.

- If the time $t$ of observability is too small.

To avoid these difficulties, we use the concept of extended spectral controllability of seeking control whose support is smaller and exactly control a finite number of specific modes of the system (1) and we study the structure of the associated spectrum. The effect of pertubation caused by such control is not estimated and its evaluation conditions the possibility to use such a method to control the state of distributed systems. 
Thus, the objective of this work is to prove that at a certain time depending on the number of controlled and support modes of control, the perturbation is inversely proportional to the time control and the spectrum is quasi symetric. A consequence of this result is that for the initial data in $L^{2}(\Omega) \times H^{-1}(\Omega)$ and an open non-empty $\omega$ of the domain $\Omega$, there is an internal control class $C^{\infty}$ which brings the system to as near state as we want a state considered in $L^{2}(\Omega) \times H^{-1}(\Omega)$.

\subsection{Reminders on the Spectral Theory}

Consider the homogeneous wave equation

$$
\left\{\begin{array}{c}
\varphi^{\prime \prime}-\Delta \varphi=0 \text { in } Q_{T} \\
\varphi(0)=\varphi_{0} \\
\varphi^{\prime}(0)=\varphi_{1}
\end{array}\right.
$$

and let the family $\left(w_{j}\right)$ for $\left\{j \in \mathbb{N}^{\star}\right\}$ the sequences of eigenfunctions of the operator $-\Delta \varphi$ and $\left(\lambda_{j}\right)_{j \in \mathbb{N}^{\star}}$ the sequence of eigenvalues associated rows in ascending order. If $N \in \mathbb{N}^{\star}$, posing

$$
F_{N}=\operatorname{Vect}\left(w_{j}\right)_{j \in\{1, \ldots, N\}}
$$

the vector space generated by the eigenfunctions $w_{1}, \ldots, w_{N}$. If initial conditions $\left(\varphi_{0}, \varphi_{1}\right) \in F_{N}^{2}$, then we have:

$$
\varphi_{0}=\sum_{j=1}^{N} \varphi_{0, j} w_{j} \text { and } \varphi_{1}=\sum_{j=1}^{N} \varphi_{1, j} w_{j}
$$

And the only solution of the homogeneous wave equation (2) $\varphi \in C\left(O, T ; F_{N}\right)$. Moreover, it is known by Lions[6, 7]:

$$
\varphi(x, t)=\sum_{j=1}^{N}\left[\varphi_{0, j} \cos \left(\sqrt{\lambda_{j}} t\right)+\varphi_{1, j} \frac{\sin \left(\sqrt{\lambda_{j}} t\right)}{\sqrt{\lambda_{j}}}\right] w_{j}(x)
$$

For $j \in \mathbb{N}^{\star}$, let

$$
\zeta_{j}=\cos \left(\sqrt{\lambda_{j}} t\right) w_{j}(x) \text { and } \xi_{j}=\frac{\sin \left(\sqrt{\lambda_{j}} t\right)}{\sqrt{\lambda_{j}}} w_{j}(x)
$$

Theorem 1 (Niane $[3,4])$

Let $N \in \mathbb{N}^{*}$ and the initial couple $\left(y_{0}, y_{1}\right) \in F_{N}^{2}$, it exist $T_{0}>0$ such that for all $T>T_{0}$, there exist a $C^{\infty}$ control $v$ with support in $\omega \times] 0, T[$ such that the solution $y$ of (1) verify:

1. The orthogonal projection of $\left(y(T), y^{\prime}(T)\right)$ on $F_{N}^{2}$ in $\left(L^{2}(\Omega)\right)^{2}$ is null.

2. $\left\|\left(y(T), y^{\prime}(T)\right)\right\|_{H_{0}^{1}(\Omega) \times L^{2}(\Omega)} \leq \frac{C(N, \omega)}{T}\left\|\left(y_{0}, y_{1}\right)\right\|_{H_{0}^{1}(\Omega) \times L^{2}(\Omega)}$ where $C(N, \omega)$ depend only of $\mathrm{N}$ and $\omega$.

\section{Remark 1}

Thus allow the flow of time, we can bring the system to a condition as nearly as we want its state equilibrium.

\section{Main Results: Differential Characterization and Decomposition Theorem}

\section{Theorem 2}

Let the quadratic functional $J$ defined over $F_{N} \times F_{N}$ by

$$
J\left(\varphi_{0}, \varphi_{1}\right)=\frac{1}{2} \int_{0}^{T} \int_{\omega} \varphi^{2}(x, t) d x d t+\left(y_{1}, \varphi_{0}\right)-\left(y_{0}, \varphi_{1}\right)
$$

where $\varphi$ is the solution of (1) for the initial data $\left(\varphi_{0}, \varphi_{1}\right)$, has a unique minimum attained at a point $\left(\psi_{0}, \psi_{1}\right)$ caracterised by

$$
J^{\prime}\left(\varphi_{0}, \varphi_{1}\right)\left(\psi_{0}, \psi_{1}\right)=\int_{0}^{T} \int_{\omega} \varphi(x, t) \psi(x, t) d x d t+\left(y_{1}, \psi_{0}\right)-\left(y_{0}, \psi_{1}\right)=0
$$

for all $\psi$ solution of (2) corresponding at initial data $\left(\psi_{0}, \psi_{1}\right) \in F_{N} \times F_{N}$.

Proof.

Functional $J$ is convex and differentiable, so if it admits a minimum, it is characterized by equation (8). So just to show that (8) has a unique solution. 
To do this, we can divide the relation (2.7) by $T$ (It may be noted that this division is not essential to show existence and uniqueness).

Let $i \in\{1, \ldots, N\}$ and $j \in\{1, \ldots, N\}$ and posing:

$$
\begin{gathered}
a_{i j}=\frac{1}{T} \int_{0}^{T} \cos \left(\sqrt{\lambda_{i}} t\right) \cos \left(\sqrt{\lambda_{j}} t\right) d t \int_{\omega} w_{i} w_{j} d x \\
a_{i, j+N}=a_{j+N, i}=\frac{1}{T \sqrt{\lambda_{j}}} \int_{0}^{T} \cos \left(\sqrt{\lambda_{i}} t\right) \sin \left(\sqrt{\lambda_{j}} t\right) d t \int_{\omega} w_{i} w_{j} d x \\
a_{i+N, j+N}=\frac{1}{T \sqrt{\lambda_{i}} \sqrt{\lambda_{j}}} \int_{0}^{T} \sin \left(\sqrt{\lambda_{i}} t\right) \sin \left(\sqrt{\lambda_{j}} t\right) d t \int_{\omega} w_{i} w_{j} d x
\end{gathered}
$$

where $\left(w_{i}\right)$ form a Hilbert basis of eigenvectors.

Let us define the matrix of rigidity $A=\left(a_{i j}\right)_{1 \leq i, j \leq 2 N}$; a square matrix of order $2 N$ then the euclidean matrix norm:

$\|A\|\|=\| A \|_{2}=\left[\sum_{1 \leq i, j \leq 2 N} a_{i j}^{2}\right]^{\frac{1}{2}}$.

Let us rewrite the relation (8) under the following conditions:

1. $\psi_{0}=w_{i}$ and $\psi_{1}=0$, which gives

$$
\sum_{j=1}^{N}\left(a_{i j} \varphi_{0 j}+a_{i, j+N} \varphi_{1 j}\right)=-\frac{1}{T} y_{1, i}
$$

2. $\psi_{0}=0$ and $\psi_{1}=w_{i}$, which gives

$$
\sum_{j=1}^{N}\left(a_{i+N, j} \varphi_{0 j}+a_{i+N, j+N} \varphi_{1 j}\right)=\frac{1}{T} y_{0, i}
$$

The relations (12) and (13) form the linear system of order $2 N$ :

$$
A \Phi=\frac{1}{T} . Y
$$

where $\Phi=\left(\varphi_{0,1}, \varphi_{0,2}, \ldots, \varphi_{0, N}, \varphi_{1,1}, \varphi_{1,2}, \ldots, \varphi_{1, N}\right)$,

$Y=\left(-y_{1,1},-y_{1,2}, \ldots,-y_{1, N}, y_{0,1}, y_{0,2}, \ldots, y_{0, N}\right)$ and $\mathrm{A}$ is the symmetric matrix whose coefficients are defined by (12) and (13).

And we remark that $\forall i, j \in\{1, \ldots, N\}$,

$$
\left\{\begin{array}{c}
a_{i, j}=\frac{1}{T}\left(\zeta_{i}, \zeta_{j}\right) \\
a_{i, j+N}=\frac{1}{T}\left(\zeta_{i}, \xi_{j}\right) \\
a_{i+N, j+N}=\frac{1}{T}\left(\xi_{i}, \zeta_{j}\right)
\end{array}\right.
$$

where (., .) denotes the scalar product in $L^{2}(\omega \times[0, T])$.

Therefore, A is a Gram matrix; Knowing that the system $\left\{\zeta_{i}\right\} \cup\left\{\xi_{i}\right\}$ for $i \in\{1, \ldots, N\}$ form a free family, we deduce that the matrix A is positive definite, so reversible. So, the system (14) admits a unique solution.

Hence the existence and uniqueness of the minimum of the functional $J$

\subsection{Decomposition Theorem}

Now, let's get down to give a representation of the solution of the linear system (14).

Let $\mathrm{D}$ be the diagonal square matrix of order $2 N$ defined as follows

$$
\left\{\begin{array}{c}
D=\left(d_{i j}\right)_{1 \leq i, j \leq 2 N} \text { with } \\
d_{i i}=\int_{\omega} w_{i}^{2} d x \text { and } \\
d_{i+N, i+N}=\frac{1}{\lambda_{i}} \int_{\omega} w_{i}^{2} d x, \forall i=1, \ldots, N
\end{array}\right.
$$




\section{Theorem 3}

1. The matrix A (14) is writen in the form

$$
A=\frac{1}{2}\left[\frac{1}{T} B+I\right]+D
$$

where $B$ is a matrix of which the euclidean norm is majored by a constant $R_{N}$ independant of $T$ and I the unit matrix.

2. There exist a constant $T_{0}>0$ such that for all $T>0$, the solution of the linear system (14) is writen: $\Phi=v_{1}+v_{2}$ with

$$
v_{2}=2 \Sigma_{k=1}^{\infty} \frac{1}{T^{k+1}}\left[-D^{-1} B\right]^{k} D^{-1} y
$$

and

$$
v_{1}=\left(v_{0,11}, v_{0,12}, \ldots, v_{0,1 N}, v_{1,11}, v_{1,12}, \ldots, v_{1,1 N}\right)
$$

where

$$
\left\{\begin{array}{l}
v_{0,1 i}=\frac{-2}{T \int_{\omega} w_{i}^{2}(x) d x} y_{1 i} \\
v_{1,1 i}=\frac{2 \lambda_{i}}{T \int_{\omega} w_{i}^{2}(x) d x} y_{0 i}
\end{array}\right.
$$

Proof.

$\forall i, j \in\{1, \ldots, N\}$ we obtain by simple calculations the components of the stiffness matrix:

$$
a_{i, i}=\left(\frac{1}{2}+\frac{\sin \left(2 \sqrt{\lambda_{i}} T\right)}{\sqrt{\lambda_{i}} T}\right) \int_{\omega} w_{i}^{2} d x
$$

- If $i \neq j$, then we have:

$$
a_{i, j}=\left(\frac{\sin \left(\sqrt{\lambda_{i}}+\sqrt{\lambda_{j}}\right) T}{2\left(\sqrt{\lambda_{i}}+\sqrt{\lambda_{j}}\right) T}+\frac{\sin \left(\sqrt{\lambda_{i}}-\sqrt{\lambda_{j}}\right) T}{2\left(\sqrt{\lambda_{i}}-\sqrt{\lambda_{j}}\right) T}\right) \int_{\omega} w_{i} \cdot w_{j} d x
$$

- Then, we have

$$
a_{i, i+N}=\frac{1}{2 \lambda_{i} T}\left(1-\cos \left(2 \sqrt{\lambda_{i}} T\right)\right) \int_{\omega} w_{i}^{2} d x
$$

- Also, if $i \neq j$, we have

$$
a_{i, j+N}=\frac{1}{2 \sqrt{\lambda_{j}} T}\left(\frac{\cos \left(\sqrt{\lambda_{i}}-\sqrt{\lambda_{j}}\right)}{\left(\sqrt{\lambda_{i}}-\sqrt{\lambda_{j}}\right) T}+\frac{\cos \left(\sqrt{\lambda_{i}}+\sqrt{\lambda_{j}}\right) T}{\sqrt{\lambda_{i}}+\sqrt{\lambda_{j}}}-\frac{2 \sqrt{\lambda_{j}}}{\lambda_{i}-\lambda_{j}}\right) \int_{\omega} w_{i} \cdot w_{j} d x
$$

- Finally,

$$
a_{i+N, i+N}=\left(\frac{1}{2 \lambda_{i}}-\frac{\sin \left(2 \sqrt{\lambda_{i}} T\right)}{4 \lambda_{i}^{\frac{3}{2}} T}\right) \int_{\omega} w_{i}^{2} d x
$$

- At least $i \neq j$, we have

$$
a_{i+N, j+N}=\frac{1}{\sqrt{\lambda_{j}} \sqrt{\lambda_{i}} T}\left(\frac{\sin \left(\left(\sqrt{\lambda_{i}}-\sqrt{\lambda_{j}}\right) T\right)}{\sqrt{\lambda_{i}}-\sqrt{\lambda_{j}}}+\frac{\sin \left(\left(\sqrt{\lambda_{i}}+\sqrt{\lambda_{j}}\right) T\right)}{\sqrt{\lambda_{i}}+\sqrt{\lambda_{j}}}\right) \int_{\omega} w_{i} \cdot w_{j} d x
$$


Of the various components of the matrix A is deducted 1. of Theorem

For the 2. of Theorem, it will suffice to prove that

$$
\left|\frac{1}{T} D^{-1} B\right|<1 \text { ie } \frac{\| D^{-1}|||B| \mid}{T}<1
$$

To do this, pose the constant

$$
T_{0}=\left\|R_{N}\right\|\left\|D^{-1}\right\|
$$

where $R_{N}=\left\|D^{-1}\right\|\|B\|$;

And for $T>T_{0}$, according to Von Neuman Lemma (see Brezis [1]), we deduce that:

$$
\left(I+\frac{1}{T} D^{-1} B\right)^{-1}=I+\sum_{k=1}^{\infty} \frac{1}{T^{k}}\left[-D^{-1} B\right]^{k}
$$

Hence the solution $\Phi$ of the linear system (14) is written as

$$
\Phi=2\left(I+\sum_{k=1}^{\infty} \frac{1}{T^{k}}\left[-D^{-1} B\right]^{k}\right) \frac{1}{T} D^{-1} y
$$

We deduce the 2. of Theorem

3. Numerical approaches to estimates for extended spectral controllability Consider the plan regular domain $\Omega$ following: Let us return to the linear system (14) of order $2 N$ where A is the stiffness matrix, $\Phi$ the unknown vector, $T$ time



Figure 1. Regular plan domain

observability and $Y$ the second member vector.

Consider the domain $\Omega$ see fig1 an open area of $\mathbb{R}^{2}$ with a regular border.

The finite element method we'll use allows us to achieve high levels of convergence with the Free-fem ++ software; Once the triangulation is made, the assembly of the stiffness matrix and the second member vector set, we can obtain estimates related to increases standards for extended internal spectral controllability of the wave equation. 3.1 Variational Formulation Let $\left(\mathcal{T}_{h}\right)$ a regular triangulation of the domain $\Omega_{h}$ into triangles of diameter less than h, the positive and constant step tending to 0 .

We define the space $W_{h}$ approximate solutions by:

$$
W_{h}=\left\{v_{h} \in V_{h} ;\left(v_{h}\right)_{\mid K} \in P_{1}(K), \forall K \in \mathcal{T}_{h}\right\}
$$

And, thereafter we define the discrete problem:

Find $u_{h} \in W_{h}$ such that: $\forall i, j \in\{1, \ldots, N\}$ 
i.

$$
a_{i, i}\left(u_{h}, u_{h}\right)=\left(\frac{1}{2}+\frac{\sin \left(2 \sqrt{\lambda_{i}^{h}} T\right)}{\sqrt{\lambda_{i}^{h}} T}\right) \int_{\omega} u_{i, h}^{2} d x
$$

ii. If $i \neq j$, then

$$
a_{i, j}\left(u_{h}, v_{h}\right)=\left(\frac{\sin \left(\sqrt{\lambda_{i}^{h}}+\sqrt{\lambda_{h}^{h}}\right) T}{2\left(\sqrt{\lambda_{i}^{h}}+\sqrt{\lambda_{j}^{h}}\right) T}+\frac{\sin \left(\sqrt{\lambda_{i}^{h}}-\sqrt{\lambda_{h}^{h}}\right) T}{2\left(\sqrt{\lambda_{i}^{h}}-\sqrt{\lambda_{j}^{h}}\right) T}\right) \int_{\omega_{h}} u_{i, h} \cdot v_{j, h} d x
$$

iii. Afterwards,

$$
a_{i, i+N}\left(u_{h}, v_{h}\right)=\frac{1}{2 \sqrt{\lambda_{i}^{h}} T}\left(1-\cos \left(2 \sqrt{\lambda_{i}^{h}} T\right)\right) \int_{\omega_{h}} u_{i, h}^{2} d x
$$

iv. Also,

$$
a_{i, i+N}\left(u_{h}, v_{h}\right)=\frac{1}{2 \sqrt{\lambda_{i}^{h}} T}\left(1-\cos \left(2 \sqrt{\lambda_{i}^{h}} T\right)\right) \int_{\omega_{h}} u_{i, h}^{2} d x
$$

v. At last, if $i \neq j$

$$
a_{i, j+N}\left(u_{h}, v_{h}\right)=\frac{1}{2 \sqrt{\lambda_{j}^{h}} T}\left(\frac{\cos \left(\left(\sqrt{\lambda_{i}^{h}}-\sqrt{\lambda_{j}^{h}}\right)\right)}{\left(\sqrt{\lambda_{i}^{h}}-\sqrt{\lambda_{j}^{h}}\right) T}+\frac{\cos \left(\left(\sqrt{\lambda_{i}^{h}}+\sqrt{\lambda_{j}^{h}}\right) T\right)}{\left(\sqrt{\lambda_{i}^{h}}+\sqrt{\lambda_{j}^{h}}\right)}-\frac{2 \sqrt{\lambda_{j}^{h}}}{\lambda_{i}^{h}-\lambda_{j}^{h}}\right) \int_{\omega_{h}} u_{i, h} \cdot v_{j, h} d x
$$

vi. Also,

$$
a_{i+N, i+N}\left(u_{h}, v_{h}\right)=\left(\frac{1}{2 \lambda_{i}^{h}}-\frac{\sin \left(2 \sqrt{\lambda_{i}^{h}} T\right)}{4 \lambda_{i}^{h, \frac{3}{2}} T}\right) \int_{\omega_{h}} u_{i, h}^{2} d x
$$

vii. At last if $i \neq j$,

$$
a_{i+N, j+N}\left(u_{h}, v_{h}\right)=\frac{1}{\sqrt{\lambda_{i}^{h}} \sqrt{\lambda_{j}^{h}} T}\left(\frac{\sin \left(\left(\sqrt{\lambda_{i}^{h}}-\sqrt{\lambda_{j}^{h}}\right)\right)}{\left(\sqrt{\lambda_{i}^{h}}-\sqrt{\lambda_{j}^{h}}\right)}+\frac{\sin \left(\left(\sqrt{\lambda_{i}^{h}}+\sqrt{\lambda_{j}^{h}}\right)\right)}{\left(\sqrt{\lambda_{i}^{h}}+\sqrt{\lambda_{j}^{h}}\right)}\right) \int_{\omega_{h}} u_{i, h} \cdot v_{j, h} d x
$$

By assembling the various components of (i.) relations to (vi.), one draws the stiffness matrix A.

Let $Y_{h}$ the second member of the discrete space $\mathcal{W}_{h}$ defined by:

$$
Y_{h}=\left(-y_{1,1}^{h},-y_{1,2}^{h}, \ldots,-y_{1, N}^{h}, y_{0,1}^{h}, y_{0,2}^{h}, \ldots, y_{0, N}^{h}\right)
$$

And, $f_{k}^{h}(s)=\int_{\Omega} f^{h}(x, s) \omega_{k}^{h}(x) d h=\int_{\Omega} \Phi^{h}(x, s) \omega_{k}^{h}(x) d h$ 


\subsection{Mesh Viewing}

Considering the regular domain $\Omega$ meshed below:
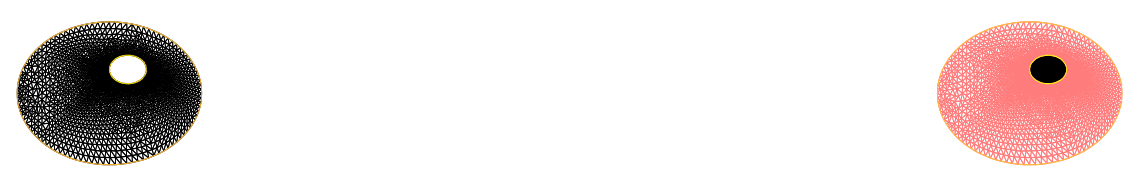

Figure 2. Refined mesh without $\omega$

Figure 3. Refined mesh with $\omega$

And so:

1. After assembly of the stiffness matrix and the second member vector, Freefem++ allows us to determine the spectrum of the rigidity matrix. And also determining the values approximate coefficients (eigenvalues) and solutions of the associated linear system.

2. In the domain $\Omega$ set (figure 2 and 3), by restricting to a portion of $\omega$ of $\Omega$ and an internal control, we see that for values below the caused disturbance is inversely proportional to the duration of the control $t$ on a fixed interval ]0,T]; Hence the values obtained are quasi-symmetric numerically relative to the values of each sets of eigenvalues see fig. 4 to fig. 8 .

\subsection{Mesh Size Characteristics are as Follows}

Table 1. Characteristics tested meshes

\begin{tabular}{|c|c|c|c|}
\hline Parameter $N$ & 100 & 200 & 300 \\
\hline Number of Triangles $N T$ & 1176 & 3582 & 4900 \\
\hline Number of nodes $N N$ & 639 & 1891 & 2501 \\
\hline
\end{tabular}

\subsection{The Spectra}

And the spectra is in the following table

Table 2. The spectra of solutions

\begin{tabular}{|c|c|c|c|c|c|}
\hline Spectrum 1 & 2 & 5 & 5 & 8 & 10 \\
\hline Spectrum 2 & 10 & 13 & 13 & 17 & 17 \\
\hline Spectrum 3 & 18 & 20 & 20 & 25 & 25 \\
\hline Spectrum 4 & 26 & 26 & 29 & 29 & 32 \\
\hline Spectrum 5 & 34 & 34 & 41 & 41 & 50 \\
\hline
\end{tabular}

Remark 3 The approximation errors in the calculation of the eigenvalues associated with this domain $\left(\Omega_{h}\right)$ range from $-8.953 .10^{-14}$ and $+4.13202 .10^{-14}$ with Freefem ++ Software. 


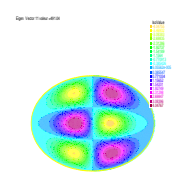

Figure 4. Spectrum 1

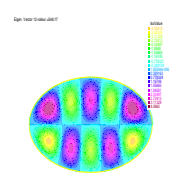

Figure 6. Spectrum 3



Figure 8. Spectrum 5
Figure 5. Spectrum 2



Figure 7. Spectrum 4

\subsection{Graphical Representation of Spectra}

\section{Numerical Interpretations}

- All eigenvalues obtained in all spectra are ranked in ascending order as demonstrated by the spectral theory. But the symmetry of the spectrum is not linked to the rank of a fixed eigenvalue.

- Numerical simulations amply illustrate two types of almost or quasi-symmetric spectra.

- And, this confirms the virtual symmetry of each spectrum recorded in Table 2 by an eigenvalue.

- Moreover, it can be seen that even by further refining the mesh and by increasing the number of series of eigenvalues obtained, the observations remain globally the same.

\section{Conclusion}

Thus, the aim of this work was to prove that from a certain time depending on the number of eigen modes controlled and the support of the control, the perturbation is inversely proportional to the control time. A consequence of this result is that for appropriately selected initial data, there is an internal control over a portion of the considered domain to a near state that we wants. And, the theoretical results obtained have been proved numerically.

\section{Acknowledgements}

The authors would like to thank the Referee for his comments and suggestions.

The authors thank the CEA-MITIC housed at the UFR SAT of Gaston Berger University of Saint-Louis. 


\section{References}

Allaire, G. (2006). Analyse numérique et optimisation. Editions Ellipses, Paris . Version sans image et avec bandeau pour usage personnel et non reproductible disponible à l'adresse: http://www.cmap.polytechnique.fr/allaire/livre2.html.

Ervedoza, S. (2009). Spectral conditions for admissibility and observability of wave système: applications to finite element method scheme. Numer.Math., 113(3), 377-415.

Ervedoza, S., \& Zuazua, E. (2008). The wave equation: Control and Numerics, ESF Research Networking Program OPTPDE and Grant MTM of the MICINN, Spain.

FreeFem++, (2015). Third edition, Version 3.32, F. Hecht, S. Auliac, O. Pironneau, Laboratoire J. L. Lions, Université Pierre et Marie Curie, Paris.

Haïm, B. (1983). Analyse fonctionnelle, Collection Mathématiques Appliquées pour la Maîtrise.Théorie et applications. Masson, Paris, xiv + 234, $2-225-77198-7,46-01(47-01), 697382(85 a: 46001)$.

Lions, J.-L. (1988). Contrôlabilité exacte, perturbations et stabilisation de systèmes distribués.Tome 2, Recherches en Mathématiques Appliquées, Research in Applied Mathematics,Volume 9, Perturbations, Masson, Paris.

Lions, J.-L. (1988). Contrôlabilité exacte, perturbations et stabilisation de systèmes distribués. Tome 1,Recherches en Mathématiques Appliquées ,Research in Applied Mathematics,Volume 8, Paris.

Niane, M. T. (1990). Régularité, contrôlabilité exacte et contrôlabilité spectrale de l'équation des ondes et de l'équation des plaques vibrantes, Thèse de Doctorat d'Etat, Université Cheikh Anta Diop de Dakar, Sénégal.

Niane, M. T. (1989). Contrôlabilité spectrale élargie des systèmes distribués par une action sur une partie analytique arbitraire de la frontière, C.R. Acad. Sci. Paris, t. 309, Série 1, 335-340.

Puel, J. P. (2008). Contrôle et équations aux dérivées partielles: Aspects de la théorie du contrôle, p. 169-188, Ed. Éc. Polytech., Palaiseau.

Seck, C., Bayili, G., Sène, A., \& Niane, M. T. (2011). Contrôlabilité exacte de l'équation des ondes dans des espaces de Sobolev non réguliers pour un ouvert poygonal, Afrika Matematika.

\section{Copyrights}

Copyright for this article is retained by the author(s), with first publication rights granted to the journal.

This is an open-access article distributed under the terms and conditions of the Creative Commons Attribution license (http://creativecommons.org/licenses/by/4.0/). 